\title{
Performance Analysis of the Gradient Feature and the Modified Direction Feature for Off-line Signature Verification
}

\author{
Vu Nguyen*, Yumiko Kawazoe ${ }^{\dagger}$, Tetsushi Wakabayashi ${ }^{\dagger}$, Umapada Pal $^{\ddagger}$, and Michael Blumenstein* \\ * School of Information and Communication Technology \\ Griffith University, Gold Coast, Australia \\ Email:vu.nguyen2@griffithuni.edu.au,m.blumenstein@griffith.edu.au \\ ${ }^{\dagger}$ Graduate School of Engineering, Mie University, TSU, Mie 514-8507, Japan \\ Email: yumiko@hi.info.mie-u.ac.jp,waka@hi.info.mie-u.ac.jp \\ ${ }^{\ddagger}$ CVPR Unit, Indian Statistical Institute, Kolkata-108, India \\ Email: umapada@isical.ac.in
}

\begin{abstract}
Feature extraction is an important process in offline signature verification. In this work, the performance of two feature extraction techniques, the Modified Direction Feature (MDF) and the gradient feature are compared on the basis of similar experimental settings. In addition, the performance of Support Vector Machines (SVMs) and the squared Mahalanobis distance classifier employing the Gradient Feature are also compared and reported. Without using forgeries for training, experimental results indicated that an average error rate as low as $\mathbf{1 5 . 0 3 \%}$ could be obtained using the gradient feature and SVMs.
\end{abstract}

Keywords-off-line signature verification; modified direction feature; gradient feature; support vector machines; Mahalanobis classifier;

\section{INTRODUCTION}

Automatic verification of signatures, a behavioral biometric, can be performed using a tablet with a stylus or using the signature scanned image. The former is called online verification and the latter is called off-line verification. Compared to online verification, which has already had commercial applications, offline signature verification is considered much harder due to the unavailability of dynamic information such as stroke order, velocity, or local pressure. Whilst on-line verification has already been used widely, the accuracy of off-line systems is still limited.

Off-line verification has several advantages over its online counterpart. Firstly, it has widely been accepted in the society. Secondly, it is more convenient as it does not require any special instruments. Thirdly, with the large amount of bank checks, credit card authorization forms, or legal documents still being signed everyday, off-line verification has considerable commercial potential.

Unlike other physiological biometrics, the characteristic of an individual's signature can only be established using an appropriate number of signature specimens. Since human signatures can vary over time, too few samples will increase the false rejection rate (FRR) of genuine signatures whilst too many samples will have the reverse effect of increasing the false acceptance rate (FAR) [1]. The collection of signatures from a large population for scientific research is not only labour intensive but also requires that the forgers are in possession of certain imitation skills. Besides, the privacy aspect of signatures discourage many from releasing their signatures to the public. Consequently, only a small number of signature databases are made accessible for scientific research.

The fact that each corpus has its own collection protocol further complicates the performance comparison of different feature extraction techniques. Preferably, the signatures of the same writer should be collected in different writing sessions. However, the organization of multiple writing sessions becomes too costly so larger signature databases, such as the GPDS [2] signature corpus, prefer the single writing session approach. In some databases, the forgers produced the forgeries after examining just one specimen. The exposure of more than one specimen to a forger encourages the forger to examine the signatures in detail to find out unique characteristics from the given specimens.

In this work, we compare the performance of two feature extraction techniques, the Modified Direction Feature [3] and the gradient feature [4], [5], using a public database with similar experimental settings. The performance of Support Vector Machines and the squared Mahalanobis distance classifiers in conjunction with the gradient feature are also reported.

The remainder of this paper is organized as follows: The next section presents the feature extraction techniques investigated in this research. After that, the squared Mahalanobis distance and SVM classification techniques are briefly mentioned in Section III. The GPDS signature corpus, which was employed in this research, is described in Section IV. Section V details the experimental settings whilst results and discussions are given in Section VI. Finally, Section VII concludes the paper and details future work. 


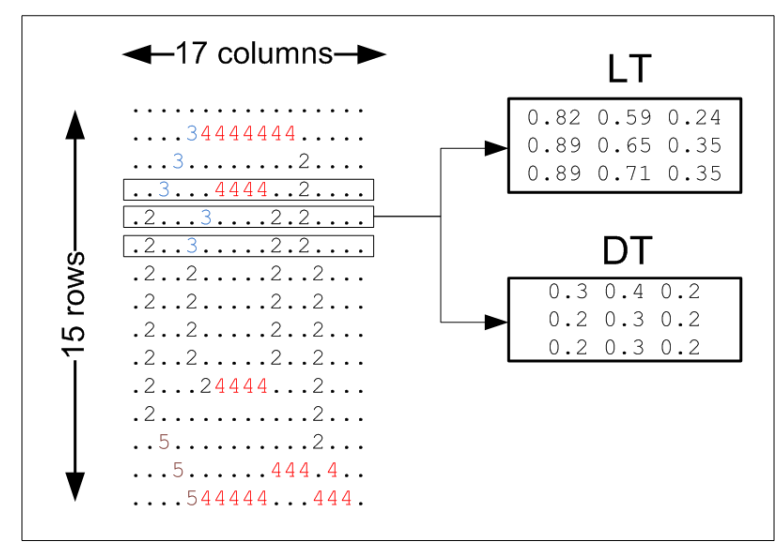

Figure 1. The LT and DT values of some rows obtained from the left to right traversal direction

\section{FEATURE EXTRACTION}

Two feature extraction techniques compared in this research are the Modified Direction Feature and the gradient feature. Both techniques utilise the directional and relative position information extracted from the input pattern.

\section{A. The Modified Direction Feature-based Feature Set}

The Modified Direction Feature, or MDF in short, is created by combining the location of transitions (LT) [6] and the direction of transitions (DT) [7] in an image pattern. Experimental results indicate that the MDF often produces a better recognition accuracy than either LT-based or DT-based features if used individually.

The MDF extraction proceeds as follows: from the preprocessed black and white signature images, the contours are first extracted. Directional codes are then assigned to each contour pixel as follows: 2 for vertical, 3 for right diagonal, 4 for horizontal and 5 for left diagonal. The code of the dominant direction in each computed contour segment is then reassigned for all the pixels of the segment. The location of transition values (LTs) are calculated as the proportion between the relative positions of each transition and the image width or height. The LT and DT values are only recorded for up to 3 transitions along each of four directions: left-right, right-left, top-down, bottom-up. The obtained LT and DT values are then assembled into 5 groups for each traversal direction before being averaged to create a feature vector with $2 \times 3 \times 4 \times 5=120$ components. The number of transitions as well as groups has been determined experimentally from previous investigations [3]. Other global features [8] including the aspect ratio, energybased feature, and maxima feature are finally incorporated to obtain a feature set with 127 feature values in total. Fig.
1 illustrates this process.

\section{B. Computation of gradient feature}

The gray-scale local-orientation histogram of the component is used for 576 dimensional feature extraction. To obtain 576-dimensional gradient-based feature vector, the following steps are executed.

Step 1: A $2 \times 2$ mean filtering is applied 5 times on the input image.

Step 2: The gray-scale image obtained in Step 1 is normalized so that the mean gray scale becomes zero with maximum value 1 .

Step 3: The normalized image is then segmented into $17 \times 7$ blocks. Compromising trade-off between accuracy and complexity, this block size is decided experimentally. To get the bounding box of the grey-scale image, the image is converted into two-tone using Otsu's thresholding algorithm [9]. This will exclude unnecessary background information from the image.

Step 4: A Roberts filter is then applied on the image to obtain the gradient image. The arc tangent of the gradient (direction of gradient) is quantized into 32 directions and the strength of the gradient is accumulated with each of the quantized direction. The strength of the Gradient $f(x, y)$ is defined as follows:

$$
f(x, y)=\sqrt{(\Delta u)^{2}+(\Delta v)^{2}}
$$

and the direction of gradient $\theta(x, y)$ is:

$$
\theta(x, y)=\tan ^{-1} \frac{\Delta v}{\Delta u}
$$

where

$$
\Delta u=g(x+1, y+1)-g(x, y)
$$

and

$$
\Delta v=g(x+1, y)-g(x, y+1)
$$

and $g(x, y)$ is the gray level of $(x, y)$ point.

Step 5: Histograms of the values of 32 quantized directions are computed for each of the $17 \times 7$ blocks.

Step 6: To further reduce the size of the feature vector without significantly distorting directional information, the $17 \times 7$ block directional histogram is down sampled into $9 \times 4$ blocks and 16 directions using $5 \times 5$ Gaussian (lowpass) filters. The $\sigma$ parameter of the filters was set to be 0.9 . Finally, a $9 \times 4 \times 16=576$ dimensional feature vector is obtained. Our earlier experiments indicated that the $9 \times 4$ block derived from $17 \times 7$ block configuration produce better results than the $9 \times 4$ block configuration without downsampling.

Fig. 2 illustrates a signature and its directional histogram. 


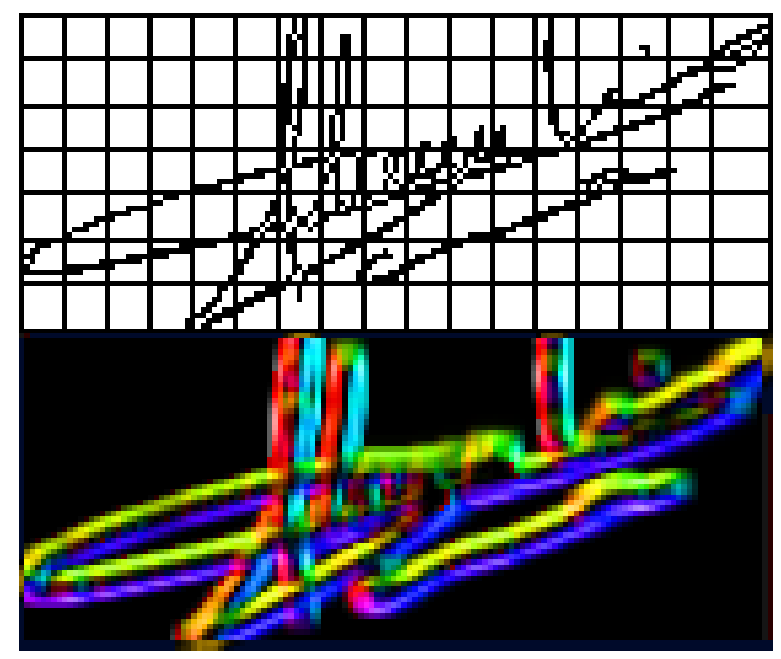

Figure 2. $17 \times 7$ blocks of a signature and its computed directional histogram

\section{Classification TeChniques}

In order to compare the performance of the reported MDF-based feature set and the gradient feature extraction technique, genuine and forged signature specimens for training and testing were selected to be similar. The two classifiers investigated in this research are Support Vector Machines (SVMs) [10] with a Gaussian kernel and the squared Mahalanobis distance. In the training phase, the classifiers were trained without using forgeries. This approach is similar to that employed by Justino et al. [1] except that the whole signature corpus was employed to construct the receiver operating characteristic (ROC) curves and find the optimal common parameters of the classifiers.

\section{A. Squared Mahalanobis Distance}

The squared Mahalanobis distance employed in this work is defined as follows:

$$
d_{M}^{2}(\mathbf{x})=\left(\mathbf{x}-\mathbf{m}_{l}\right)^{T} \Sigma_{l}^{-1}\left(\mathbf{x}-\mathbf{m}_{l}\right)
$$

where $x$ is the feature vector generated from an input image; $\mathbf{m}_{l}$ and $\Sigma_{l}$ respectively are the mean and the covariance matrix of the training vectors of writer $l$. If the output value $d^{2}(\mathbf{x})$ of this classifier is less than a predefined threshold value, the input signature is judged as genuine, otherwise it is a forgery.

\section{B. Support Vector Machines}

The other classification technique employed in this research is the SVM approach developed by Vapnik [11]. SVMs have been successfully applied in a wide range of applications. Originally, SVMs were designed for two class problems. They look for the optimal hyper-planes which maximize the distance, or margin, between two classes. This hyper-plane is characterized by the normal vector, which can be expressed as a linear combination of the nearest examples of both classes, named support vectors.

In order to extend SVMs for much more general decision surfaces than hyper-planes, kernel techniques are used. Original data is embedded into a high dimensional space, $\mathrm{H}$ (Hilbert Space), using a particular mapping. The inner product in $\mathrm{H}$ is computed by the kernel function: $\kappa(x, y)=\phi(x) \cdot \phi(y)$ which must satisfy Mercer's Condition. Some popular kernel functions can be listed are Polynomial kernels, Sigmoid kernels, Gaussian kernels etc. The Gaussian kernel has been employed and investigated in this research as it often produced better results in our earlier investigations [8]. Due to the popularity of the SVM, its details will not be expanded upon in this paper.

\section{GPDS SIGNATURE CORPUS}

GPDS-160 [12] is an earlier release of the much larger GPDS-960 [2] signature corpus which consists of 160 sets of signatures. This signature corpus can be obtained upon request at the URL: http://www.gpds.ulpgc.es/download/index.htm. Each signature set of this corpus consists of 24 genuine signatures and 30 simple forgeries. Genuine signatures of the same set were collected in a single writing session. Ordinary people were employed as forgers for the creation of the forgeries. Each forger was asked to produce 3 forgeries for each writer of a set of 5 randomly chosen writers. Before producing the forgeries for a specific writer, a forger was shown 5 random genuine signatures and allowed unlimited time to practise. Since the forgers were not experts, their products were considered simple forgeries. All the signatures and forgeries were scanned as 256 gray level images at the resolution of 300 dpi before being converted into black and white images using Otsu's thresholding algorithm.

\section{EXPERIMENTAL SetTings}

For every signature set, the classifier was trained using 12 genuine signatures and 400 random forgeries. No simple forgeries were used to train the classifier. The random forgeries are the genuine specimens taken from 100 other sets, four signatures per set. The testing process employed the 12 remaining genuine signatures and 15 forgeries. This process was repeated 30 times in order to produce a reliable result for both training and testing within class specimen ids regenerated randomly each time. These experimental settings as well as sample ids are identical to those described in [8] excepts that the training of the squared Mahalanobis distance did not employ random forgeries. The training and testing process is performed with different value of gamma in the experiments using SVM and with different threshold values in the experiments using squared Mahalanobis distance. For each classifier, the averaged False Rejection Rate and the False Acceptance Rate were calculated and the ROC curves were plotted. 
Table I

COMPARATIVE EXPERIMENTAL RESULTS OF DIFFERENT CLASSIFIERS

\begin{tabular}{|c|c|c|c|c|}
\hline Feature & Classifier & FAR & FRR & AER \\
\hline MDF & SVM & $17.25 \%$ & $17.25 \%$ & $17.25 \%$ \\
\hline Gradient & SVM & $16.54 \%$ & $13.51 \%$ & $15.03 \%$ \\
\hline Gradient & $d_{M}^{2}(\mathbf{x})$ & $14.80 \%$ & $18.63 \%$ & $16.52 \%$ \\
\hline
\end{tabular}

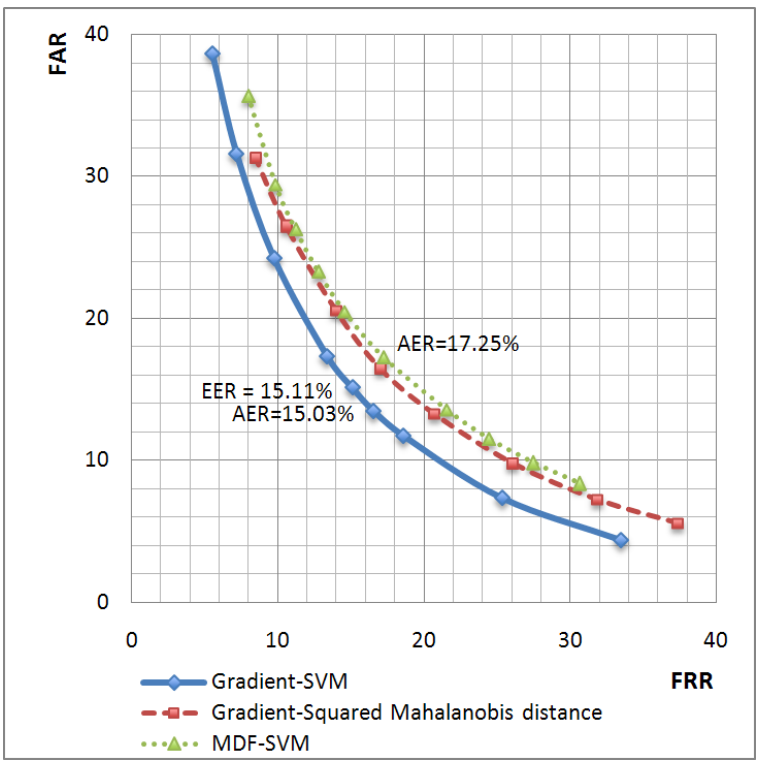

Figure 3. The ROC curves of the MDF and gradient feature obtained using SVM and squared Mahalanobis distance. Employing SVMs, the gradient feature produced the lowest error rates.

\section{RESUlts AND Discussions}

In the experiments using the gradient feature, the squared Mahalanobis distance classifier produced an average error rate (AER) of $16.52 \%$ and an equal error rate (EER) of $16.77 \%$. When SVMs were employed, the gradient feature produced the lowest AER of $15.03 \%$ and the EER of $15.11 \%$. The FAR and FRR respectively are $16.54 \%$ and $13.51 \%$ at the best AER. In comparison to other researches using the GPDS-160 corpus, these results of gradient feature are very encouraging. Employing simple forgeries for training, Ferrer et al. [12] and Vargas et al. [13] respectively obtain the AER of $13.35 \%$ and $12.33 \%$.

From the obtained experimental results, it is also observed that the the performance of the squared Mahalanobis distance classifier are very encouraging, compared to those obtained using SVMs. Table 1 summarises the key results of this research.

The gradient feature outperforms the previously reported results of the MDF-based feature set whose lowest AER obtained using SVMs was $17.25 \%$. This could be partly explained by the gradient feature's significantly larger feature vector of 576 dimensions, nearly 5-fold the size of MDF, which effectively describe a signature. Fig. 3 presents the
ROC curves of the gradient feature obtained using SVM and squared Mahalanobis distance classifiers.

\section{CONCLUSIONS AND FUture WORK}

Performance comparison is important in scientific research. However, in the area of off-line signature verification, fair comparison is difficult due to the unavailability of a standard signature corpus.

In this work, two comparisons have been made. The first one is the performance of two feature extraction techniques, the gradient feature and the Modified Direction Feature. The second one is the performance of the Support Vector Machine and the squared Mahalanobis distance classifiers in conjunction with the gradient feature.

Working together, the gradient feature and the SVM produced the best AER of $15.03 \%$ which is $2.22 \%$ better than the AER of the MDF.

As the size of the MDF feature vector is much smaller than the gradient feature, it is suggested that a larger and appropriate feature vector might improve the performance of the MDF. In future work, we will concentrate on a new grouping and averaging strategy especially in the horizontal directions as currently employed by the gradient feature. In addition, we will also investigate the performance of other classifiers, such as the least squared SVM, spiking neural network, and self organising map neural network, in conjunction with the feature extraction techniques described in this paper.

\section{REFERENCES}

[1] E. J. R. Justino, F. Bortolozzi, and R. Sabourin, "A comparison of SVM and HMM classifiers in the off-line signature verification," Pattern Recognition Letters, vol. 26, no. 9, pp. 1377-1385, 2005.

[2] F. Vargas, M. Ferrer, C. M. Travieso, and J. Alonso, "Offline handwritten signature GPDS-960 corpus," in $9^{\text {th }}$ ICDAR. IEEE Computer Society, 2007, pp. 764-768.

[3] M. Blumenstein, X. Y. Liu, and B. Verma, "A modified direction feature for cursive character recognition," in Intl. Joint Conf. on Neural Networks, 2004.

[4] T. Wakabayashi, S. Tsuruoka, F. Kimura, and Y. Miyake, "Increasing the feature size in handwritten numeral recognition to improve accuracy," Systems and Computers in Japan, vol. 26, pp. 2046-2053, 1995.

[5] M. Shi, Y. Fujisawa, T.Wakabayashi, and F. Kimura, "Handwritten numeral recognition using gradient and curvature of gray scale images," Pattern Recognition, vol. 35, pp. 20512059, 2000

[6] P. D. Gader, M. A. Mohamed, and J.-H. Chiang, "Handwritten word recognition with character and inter-character neural networks," SMC, IEEE Trans. on, Part B, vol. 27, no. 1, pp. 158-164, 1997.

[7] M. Blumenstein, B. Verma, and H. Basli, "A novel feature extraction technique for the recognition of segmented handwritten characters," in $7^{\text {th }}$ ICDAR. IEEE Computer Society, 2003, pp. 137-141.

[8] V. Nguyen, M. Blumenstein, and G. Leedham, "Global features for the off-line signature verification problem," in $10^{\text {th }}$ ICDAR. IEEE Computer Society, 2009, pp. 1300-1304. 
[9] N. Otsu, "A threshold selection method from gray-level histogram," SMC, IEEE Trans. on, vol. 9, pp. 62-66, 1979.

[10] T. Joachims, Advances in Kernel Methods - Support Vector Learning. MIT-Press, 1999, ch. Making large-Scale SVM Learning Practical.

[11] V. Vapnik, Statistical Learning Theory. New York: Wiley, 1998.
[12] M. A. Ferrer, J. B. Alonso, and C. M. Travieso, "Offline geometric parameters for automatic signature verification using fixed-point arithmetic," IEEE PAMI, Trans. on, vol. 27, pp. 993-997, 2005.

[13] J. F. Vargas, M. A. Ferrer, C. M. Travieso, and J. B. Alonso, "Off-line signature verification based on high pressure polar distribution," in $11^{\text {th }}$ ICFHR, 2008, pp. 373-378. 